\title{
FDA appointee faces angry, demoralized staff
}

$D$ resic resident Bush recently nominated Mark B. McClellan—a physician, economist, and presidential advisor on healthcare policy issues - to be commissioner of the US Food and Drug Administration (FDA; Rockville, $\mathrm{MD}$ ). If the Senate confirms this appointment, as is expected, McClellan could confront serious tensions in dealing with the FDA Center for Biologics Evaluation and Research (CBER), where many officials seem frustrated, if not outright angry, after being told abruptly early in September to cede responsibility for regulating therapeutic biologics to the agency's sister Center for Drug Evaluation and Research (CDER) (Nat. Biotechnol. 20, 960, 2002).

Although Bush's move to fill the top post at the FDA was considered long overdue, initial responses to his choice are generally welcoming, suggesting McClellan will sail through a review by the Senate, whose members are focused more on Iraq, the November elections, and unresolved legislative matters that include a series of overdue appropriations bills. Indeed, during McLellan's confirmation hearing on October 7, Senator Edward Kennedy Jr. (D-MA), who chairs the Senate Committee on Health, Education, Labor, and Pensions, and the committee's ranking Republican member, Senator Judd Gregg (R$\mathrm{NH}$ ), expressed hopes of moving quickly to confirm his appointment-explaining that doing so would help to keep the FDA "rigorous" and "on track" with its important regulatory missions, reduce approval times for new treatments, and boost sagging morale among its professional staff.

McClellan approaches this new job with an unusual mix of skills. Before heading in mid2001 to the White House to become a healthcare policy advisor and member of the Council of Economic Advisers, he served on the faculty at Stanford University (Stanford, CA) in medicine and economics, having earned doctoral degrees in both these subject areas. His research specialties include the health and economic well-being of older Americans, the economic and policy factors influencing medical treatment decisions, technological change in health care and its consequences for health and medical expenditures, "uninsurance," and the relationship between health and economic well-being.

Of course, economics is cited as the main force driving the recent transfer of regulatory responsibilities for therapeutic biologics from the CBER to the CDER. That move is "a complex process [that will] hopefully improve overall management" and is intended to bring "more consistency and economies of scale,"
McLellan said during the Senate committee hearing in October. Despite these changes, there will be no "reduction in force," he added, noting that skilled professionals will continue to be needed in both agency centers to deal with their "immense responsibilities...more effectively and efficiently." He pledged to "fill hundreds of professional positions" and said that enhancing the work environment at the FDA would be a "top priority."

Nonetheless, observers familiar with the CBER are questioning whether the recent surprise decision to transfer the review of therapeutic biotechnology products to the CDER will actually improve either consistency or regulatory throughput. "The small biotechnology companies will be hurt, because they need more guidance," says one close observer. There is also some fear that scientific thoroughness and safety might be sacrificed insofar as the CBER's remaining duties are concerned-in part because user fees supporting center programs could be siphoned over to the CDER along with the major products that are generating those fees, and also because many scientist-regulators who now work at the CBER are likely to flee but will be difficult to replace if their successors are discouraged, for the sake of heightened efficiency, from conducting research.

Such anxieties simmered near the boiling point during the September CBER centennial symposium, "Science and the Regulation of Biological Products: From a Rich History to a Challenging Future," held not long after the changes were announced. The mood among participants reflected defiance rather than what one might expect for such a celebration.

In that volume and during the symposium, CBER director Kathryn Zoon pointed repeatedly to the important "tradition" within the center of "integrating innovative science with innovative regulation." Jay Siegel, director of the Office of Therapeutics Research and Review (OTRR), whose principal operations are being moved to the CDER, outlined OTRR experiences in implementing a "sciencebased" approach to regulating therapeutic biologics during the past two decades, noting a steady reliance on "state-of-the-art input," frequent consultations with representatives of the biotechnology industry, and many additional challenges involved in ensuring safety, consistency of manufacture, and efficacy for such products.

Agency officials later provided statistics to document the regulatory performance of the CBER during the past several years, including the number of cycles to approvals, approvals within 12 months of submission, median time to approval, and frequency of meetings with industry. For example, between 1996 and 2000, the OTRR approved 14 of 22 applications for new biological therapeutics within 12 months of submission, a rate of $64 \%$. Of these 22 applications, 13 were assigned priority review schedules (10 of these were approved within 12 months) and 9 were assigned standard schedules (4 of these were approved within 12 months). In addition, "CBER has maintained an outstanding record of safety," officials note. "There has never been a need to recall an OTRR-approved biotechnology drug due to safety concerns."

"People criticize CBER as slow, and it is a pain... working with [CBER], but clinical protocols are superior to protocols of several years ago," said centennial symposium participant W. French Anderson of the University of Southern California (Los Angeles, CA), a prominent researcher in gene therapy, over which the CBER will continue to have regulatory oversight. "Breaking up CBER is wrong and short-sighted, and approaches being irresponsible."

Jeffrey L. Fox, Washington, DC

\section{Biotechs hold their own in shifting industry}

nvestors' confidence in the big drug companies is being badly shaken by the widening rift between the pharmaceutical industry and the biotech sector, according to industry analysts. The changed landscape is tempting more biotechnology firms to steer clear of what may turn out to be unprofitable partnerships with the large-cap pharmaceutical sector-provided they have the cash.

In a report "Big Pharma is Dead, Long Live Big Pharma" published in September, equity analysts UBS Warburg (London \& New York) point out that a stream of profit warnings and product failures has washed away the traditional status of pharmaceutical stocks as a defensive investment. A key reason, says UBS Warburg, is that big pharma's R\&D productivity is declining fast. From 1998 to 2000 the number of new chemical entities approved annually by the US Food and Drug Administration (FDA; Rockville, MD) was consistently in the high 